\title{
Luteal progesterone level correlated with immunotherapy success of patients with repeated implantation failures
}

\author{
Omar Sefrioui $^{1 \bullet}$, Aicha Madkour ${ }^{2 * \bullet}$, Nouzha Bouamoud ${ }^{2}$, Ismail Kaarouch ${ }^{1,2,3}$, \\ Brahim Saadani ${ }^{4}$, Saaïd Amzazi ${ }^{2}$, Henri Copin ${ }^{5}$, Moncef Benkhalifa ${ }^{5}$, Noureddine \\ Louanjli $^{3}$, with the Lorem Ipsum Consortium 9 \\ 1 Anfa Fertility center, Private Clinic of Human reproduction and endoscopic surgery, \\ Casablanca, Morocco \\ 2 Biochemistry and Immunology Laboratory, Mohammed V University in Rabat, \\ Faculty of Sciences, Rabat, Morocco \\ 3 Labomac IVF centers and clinical laboratory medicine, Casablanca, Morocco \\ 4 IVF center IRIFIV, Clinique des Iris, Casablanca, Morocco. \\ 5 Reproductive Biology and Medical Cytogenetics Laboratory, Regional University \\ Hospital and School of Medicine, Picardie University Jules Verne, Amiens, France \\ OThese authors contributed equally to this work. \\ madkouraicha.ivf@gmail.com
}

\begin{abstract}
Immunotherapy using PBMC administration demonstrated relatively its effectiveness to treat RIF patients but it still unclear to explain some miscarriages. Luteal progesterone level (LPL) issued from corpus luteum after embryo implantation stage could be informative basis data to personalize immunotherapy for RIF patients predicting clinical outcomes. This randomized controlled study included 70 patients undergoing ICSI program presenting at least 3 RIF: 39 for Control of untreated patients and 31 for PBMC-test concerning treated patients with immunotherapy. For PBMC-test group, Peripheral Blood Mononuclear Cells (PBMCs) were isolated from patients on ovulation induction day and cultured three days to be administered to intrauterine cavity of patients two days before fresh embryo transfer. LPL was analyzed at day 15 after embryo transfer and clinical outcomes were calculated including implantation, clinical pregnancy and miscarriage rates. Clinical outcomes were doubly improved after immunotherapy including implantation and clinical pregnancy rates comparing Control versus PBMC-test (10\% and $21 \%$ vs $24 \%$ and $45 \%$ ). In the other hand, this strategy showed an increase over double in LPL ( $4 \mathrm{ng} / \mathrm{ml}$ for Control vs $9 \mathrm{ng} / \mathrm{ml}$ for PBMC-test) while the latter was correlated to clinical pregnancy. Bypassing the effectiveness of this immunotherapy approach for RIF patients, it is directly correlated to LPL proving the interactive reaction between immune profile of the treated patients and progesterone synthesis by corpus luteum.
\end{abstract}

\section{Introduction}

Though in vitro fertilization (IVF) success is generally limited to $30 \%$ depending on embryo implantation, the major part of implantation establishment is bypassing embryo quality and its genetic integrity highlighting the communication between embryo and the mother. This cross talk is essentially orchestrated by hormonal and immune dialogues 
in order to assure embryo invasion in the maternal endometrium without rejecting the fetal allograft. Furthermore, it is already known that progesterone (P4) is one of the most important implantation/pregnancy success keys for its effects on the endometrium and early pregnancy survival while its removal results in miscarriage [1-3].

$\mathrm{P} 4$ is a hormonal key to modulate the maternal immune system by reducing natural killer (NK)-cell activity [4], inhibiting cytotoxic T-cell activity [5], increasing HLA-G production in trophoblast cells [6], increasing suppressor-cell levels [7] and as special mechanism, it is able to induce lymphocyte-blocking proteins production such as progesterone-induced blocking factor (PIBF) [8-11]. Generally, its anti-inflammatory effect reported by several studies showing that it is essential to modify the cytokine response from pro-inflammatory profile presented by Th1 during embryo implantation to anti-inflammatory profile presented by Th2 for pregnancy maintain [12-16,18, 19]. Thus, Th1/Th2 unbalance could explain implantation failures in some patients with RIF, RPL or recurrent miscarriages (RM) [20-22].

Indeed, with special interest on immunology of reproduction, Yoshioka et al. [23] was the first team which could to realize immunotherapy for patients with repeated IVF failures based on intrauterine administration of peripheral blood mononuclear cells (PBMC). Then, several studies developed this novel approach with some modifications on PBMC preparation protocol, including fresh or frozen cycles, embryo day 3 or blastocyst transfer, patients with at least 2, 3 or 4 RIF in order to improve significantly clinical outcomes and decrease the miscarriage rates [22,24,25].

Furthermore, PBMC immunotherapy could to be efficient for some RPL cases and avoiding miscarriages $[22,26,27]$ despite lack of clear definition toward RIF and RPL [28]. This issue led us to wonder about mechanism of PBMC immunotherapy by what it could modulate maternal immune response homing Th1/Th2 balance required for implantation and pregnancy stage in order to manage RIF and RPL clinical cases and its effect on luteal $\mathrm{P} 4$ synthesis. Indeed, it is known that P4 is an indispensable factor for endometrium decidualization and for early stage of clinical pregnancy to prepare an adequate immune environment for fetus and low LPL results abnormal ongoing pregnancy $[18,19,29-32]$.

Forward, it is increasingly clear that embryo implantation is dependent in one side on immune local mechanisms and in another side on endocrine mechanisms related to luteal P4 synthesis while their interactive reaction is still kept into question for pregnancy achievement. For this reason, while our previous work [22] was based on the implementation of PBMC immunotherapy for RIF patients and proving its efficiency tripling the clinical outcomes, the present work was more focused on demonstrating the correlation between LPL and immunotherapy success highlighting the interactive reaction between luteal P4 synthesis by CL and immune system.

\section{Materials and methods}

\section{Ethical Standards}

The study was approved by the ethics committee, (Comité d'Ethique pour la Recherche Biomédicale- Faculty of Medecine and Pharmacy, University Mohammed V, Rabat, Morocco) and patients provided written informed consent after being presented with the terms and issues of the study. The authors assert that all procedures contributing to this work comply with the ethical standards of the relevant national and institutional committees on human experimentation and with the Helsinki Declaration of 1975, as revised in 2008. 


\section{Patient's selection and study design}

This was a prospective randomized study over two years conducted in Anfa Fertility Center including 70 couples who attended IVF program with at least 3 RIF without female age limit while 48 patients of them were less than 40 years old. In the selected couples, women had unremarkable clinical history and comparable clinical features and embryological outcomes (Table 1). All women received the same antagonist ovarian stimulation protocol [22] to minimize the effect of other parameters. Indeed, the whole lot was divided into two groups; treated group with PBMC immunotherapy (PBMC-test, $\mathrm{n}=31$ ) and the control group without treatment (Control, $\mathrm{n}=39$ ).

Table 1. Comparison of the patient's characteristics.

\begin{tabular}{|c|c|c|c|}
\hline & PBMC-test $\mathbf{( n = 3 1 )}$ & Control $(\mathbf{n = 3 9})$ & $\mathbf{p}-\mathbf{v a l u e}$ \\
\hline Age of the partner & $42.81 \pm 8.48$ & $42.67 \pm 5.58$ & $0.93(\mathrm{~ns})$ \\
\hline Age of the patient & $35.81 \pm 4.89$ & $36 \pm 4.71$ & $0.82(\mathrm{~ns})$ \\
\hline Number of RIF & $5.39 \pm 1.76$ & $5.26 \pm 1.96$ & $0.77(\mathrm{~ns})$ \\
\hline AMH (ng/ml) & $2.61 \pm 1.87$ & $2.03 \pm 1.35$ & $0.13(\mathrm{~ns})$ \\
\hline Estradiol $\mathbf{( p g / m l )}$ & $32.87 \pm 23.04$ & $33.39 \pm 16.51$ & $0.91(\mathrm{~ns})$ \\
\hline Progesterone (ng/ml) & $0.48 \pm 0.22$ & $0.41 \pm 0.23$ & $0.21(\mathrm{~ns})$ \\
\hline Endomtrial thikness (mm) & $9.32 \pm 1.62$ & $9.18 \pm 2.4$ & $0.12(\mathrm{~ns})$ \\
\hline Number of oocytes per patient & $9.13 \pm 4.1$ & $8.26 \pm 5.27$ & $0.45(\mathrm{~ns})$ \\
\hline Maturation rate (\%) & $(192 / 283) 68 \%$ & $(232 / 322) 72 \%$ & $0.78(\mathrm{~ns})$ \\
\hline Cleavage rate (\%) & $(131 / 134) 98 \%$ & $(172 / 180) 96 \%$ & $0.80(\mathrm{~ns})$ \\
\hline Good quality embryos rate (A+B) & $(63 / 131) 48 \%$ & $(97 / 172) 56 \%$ & $0.78(\mathrm{~ns})$ \\
\hline Total number of embryos transferred & 67 & 79 & $0.42(\mathrm{~ns})$ \\
\hline Number of embryos transferred per patient & $2.16 \pm 0.78$ & $2.03 \pm 0.63$ & $0.42(\mathrm{~ns})$ \\
\hline
\end{tabular}

Results are expressed as $\mathrm{n}, \mathrm{n}(\%)$ or mean \pm standard deviation (SD). A statistic significant difference is considered when $\mathrm{P}<0.05$ (n). $\mathrm{P} \geq 0.05$ is not significant (ns). AMH, estradiol and progesterone were measured on day 2 of the cycle and the endometrial thickness was evaluated in day of oocyte retrieval. Cleavage rate was calculated relatively to embryos at day 3 by 2 pronucleus.

\section{IVF procedures}

Embryos produced by ICSI [22] were cultured up to day 3. Adequate embryo quality (good quality embryos; $\mathrm{A}+\mathrm{B}$ ) was defined based on the presence of uniformly sized and shaped blastomeres and fragmentation lower or equal to $10 \%$. One or two good quality embryos were transferred in utero using a Frydman catheter (CCD Laboratories, Paris, France). The implantation success (observation of the embryo sac) was assessed by ultrasound imaging and calculated relative to the number of transferred embryos. Clinical pregnancy was confirmed by ultrasound imaging 6-8 weeks after embryo transfer and calculated relative to the number of transferred cycles. The miscarriage ratio was calculated relative to the number of clinical pregnancies after the first trimester. Each couple went through a single ICSI cycle during this study.

\section{PBMC immunotherapy and LPL assay}

After antagonist ovarian stimulation protocol, a blood sample is scheduled on the day of ovulation induction to isolate PBMCs using a separation protocol based on Ficoll. PBMCs are well prepared after a culture for $72 \mathrm{~h}$ and then transferred to the patient in utero two days before embryo transfer as it was elucidated by Madkour et al. [22]. After embryo transfer, patients receive oral Utrogestan $(200 \mathrm{mg} \times 2 /$ day $)$ for luteal support. 
In the course of our study, the included patients underwent the LPL analysis at day 15 after embryo transfer to reflect the P4 synthesis by CL after implantation using the serum for the first pregnancy test for $\boldsymbol{\beta}$-hCG assay. Indeed, the LPL analysis was assessed using the immunological technique of electro-chemiluminescence (ECLIA, Roche, Mannheim, Germany) at LABOMAC center.

\section{Statistical analysis}

Data are presented as the mean \pm standard deviation (SD) or percentage of the total. Data were analyzed with the Student's t-test for comparison of mean values or with the chi squared test for comparison of percentages, and r-correlations using Statistical Package, version 6.0 (Statistica); $\mathrm{p}<0.05$ shows significant differences. Then, the mean values of each parameter's results were evaluated to calculate the study power with the post-hoc test using the $\mathrm{G}^{*}$ Power software (version 3.0.10).

\section{Results}

Clinical outcomes were doubly improved after immunotherapy including implantation and clinical pregnancy in Control versus PBMC-test for patients with at least 3 RIF ( $10 \%$ and $21 \%$ vs $24 \%$ and $45 \%$ ) while the effect of PBMC on miscarriage rate was non-significant ( $75 \%$ vs $21 \%$; $=0.06)$. In the other hand, this strategy shows an increase over double in LPL (9ng/ml for PBMC-test vs $4 \mathrm{ng} / \mathrm{ml}$ for Control) showing significant correlation with clinical pregnancy rate for PBMC treated patients (Table 2). Moreover, the LPL was not influenced by RIF number with non significant $\mathrm{r}$ correlation $(\mathrm{r}=-0.36)$

Table 2. Clinical outcomes and luteal progesterone level after PBMC immunotherapy for patients with at least 3 RIF.

\begin{tabular}{|c|c|c|c|c|}
\hline Clinical outcomes & PBMC-test $(\mathbf{n = 3 1})$ & Control $(\mathbf{n = 3 9})$ & $\mathbf{p}$-value & Power 1- $\beta$ \\
\hline Implantation rate (\%) & $(16 / 67) \mathbf{2 4 \%}$ & $(8 / 79) \mathbf{1 0 \%}$ & $\mathbf{0 . 0 2}(\mathrm{s})$ & $\mathbf{8 9 \%}$ \\
\hline Clinical pregnancy rate (\%) & $\mathbf{( 1 4 / 3 1 ) \mathbf { 4 5 \% }}$ & $(\mathbf{8} / \mathbf{3 9}) \mathbf{2 1 \%}$ & $\mathbf{0 . 0 3}(\mathrm{s})$ & $\mathbf{7 1 \%}$ \\
\hline Miscarriage rate (\%) & $(3 / 14) 21 \%$ & $(6 / 8) 75 \%$ & $0.06(\mathrm{~ns})$ & $\mathbf{9 9 \%}$ \\
\hline LPL (ng/ml) & $\mathbf{9 . 3 2} \pm \mathbf{5 . 7 0}$ & $\mathbf{3 . 8 0} \pm \mathbf{3 . 6 5}$ & $\mathbf{0 . 0 0 0 0 1}(\mathbf{s})$ & $\mathbf{9 9 \%}$ \\
\hline r-correlation (LPL to clinical pregnancy) & $\mathbf{0 . 2 8}(\mathbf{p = 0 . 0 1 )}$ & $0.16(\mathrm{p}=0.32)$ & - & - \\
\hline
\end{tabular}

Results are expressed as $\mathrm{n}, \mathrm{n}(\%)$ or mean \pm standard deviation (SD). A statistic significant difference is considered when $\mathrm{P}<0.05$ (n). $\mathrm{P} \geq 0.05$ is not significant (ns). Power $1-\beta$ ( $\beta$ is error type II) is calculated basing on difference of mean values between two groups (PBMC-test vs Control) with $\alpha=0.05$ ( $\alpha$ is error type I). Power value is considered highly important when it is above $80 \%$. r-correlation was calculated relatively to LPL depending on clinical pregnancy rate in each group of PBMC-test and Control, and it is considered significant when $\mathrm{P}<0.05$ and non significant when $\mathrm{p} \geq 0.05$. The implantation rate is expressed as the ratio between the number of embryonic sacs and the total number of transferred embryos; the miscarriage rate is expressed relative to the number of clinical pregnancies. LPL (Luteal progesterone level) was measured at day 15 after embryo transfer.

\section{Discussion}

Despite there is not clear differential clinical diagnostic to differ between RIF and RPL, over $75 \%$ of pregnancy failures are due to implantation failures [33]. Whatever controversies regarding RIF and RPL clinical definition, hypothetically in our previous study [22] it was suggested that RIF is due to pro-inflammatory (Th1) deficiency while RPL is due to Th1 persistence inhibiting the anti-inflammatory (Th2) release. Therefore, in this current study following the results of our previous work [22] PBMC 
immunotherapy was efficient for RIF patients since their second implantation failure to double their chance to conceive. Nevertheless, we are not the only team who are prescribing this kind of treatment to patients with RIF or generally with IVF failures. Yoshioka et al. were the pioneers in this approach application while the research teams followers could to involve some technical modifications in PBMC preparation protocol [23]. Some could to prove the efficiency of hCG supplementation on PBMC culture for $72 \mathrm{~h}[22,24]$ or trying to minimize latter to $24 \mathrm{~h} \quad[26,27,34]$ while others were more focused on the efficiency of $\mathrm{CRH}$ [25]. All these technical adaptations were occurred in order to enhance at maximum the function of PBMC and their cytokines secretions to activate thereafter the maternal immune system into endometrium after an intrauterine administration and be ready for embryo implantation. Indeed, as expected, implantation rate after PBMC immunotherapy was over double compared to control ( $24 \%$ vs $10 \%$; table 2 ) and the result was similar to other studies with interval $21-25 \%$ for treated patients $[22-24,26,27]$.

Nevertheless, it was commonly accepted that with insufficient P4 production causing miscarriages could be solved simply by an exogenous $\mathrm{P} 4$ administration in order to regulate the inflammatory mediators of pregnancy and even for patients undergoing IVF process in fresh or frozen cycles to improve clinical outcomes prior embryo transfer $[29,35,36]$. Indeed, P4 presents an anti-inflammatory action enhancing Th2 cytokines production to maintain pregnancy $[37,38]$.

However, our doubled clinical outcomes including implantation and clinical pregnancy rates could have more evident explanation especially when LPL showed high increase in PBMC-test compared to control $(9 \mathrm{ng} / \mathrm{ml}$ vs $4 \mathrm{ng} / \mathrm{ml})$ with positive correlation relatively to clinical pregnancy just for treated RIF patients $(\mathrm{r}=0.28)$ (Table 2). However, $4 \mathrm{ng} / \mathrm{ml}$ of LPL was not correlated to clinical pregnancy rates for RIF patients in control group $(\mathrm{r}=0.16)$. This observation allowed us to conclude that certainly even with $\mathrm{P} 4$ importance to maintain clinical pregnancy, this latter could be occurred. Moreover, it explained why $\mathrm{P} 4$ supplementation treatments kept into question their efficiency for RIF, RPL and RM patients despite the maternal immune system is dysregulated led toward Th1 or Th2 [21,29,39,40]. In the opposite side, when this latter is probably trying to turn back its balance via PBMC immunotherapy which acted not only into endometrium but also in CL to produce more the P4, LPL became correlated to clinical pregnancy. This hypothesized interactive reaction between LPL issued from CL and PBMC administrated into endometrium, is realized in order to assure the embryo implantation and pregnancy maintain homing Th1/Th2 balance(Fig 1).

Furthermore, luteal P4 would regulate the uterine level synthesis of CSF-1, cytokine essential for the vascularization of the endometrium and to maintain pregnancy by increasing just before implantation to achieve a peak tripled to day 15 of pregnancy $[41,42]$. Our results show a $63 \%$ increase in the synthesis of the luteal progesterone in pregnant patients treated with $\mathrm{PBMC}$ which joins perfectly the observed effect of PBMC on clinical pregnancy rate in our study. It seems that this effect is mediated by Th2 cells secreting IL4 and IL10 able to optimize the recruitment of leukocytes for VEGF secretion in CL [13]. The latter is better vascularized release P4 production [43].

Te PBMC effect could not be certainly over early pregnancy stage to balance maternal immune system and LPL while P4 will be placental and the ongoing pregnancy until delivery will be more influenced by fetus and genetic reproductive function. May be for this reason, PBMC immunotherapy is less effective to avoid miscarriages as showed in our study with non-significant difference in miscarriage rate (21\% for PBMC-test and $75 \%$ for control; Table 2) and confirmed by others [22, 26].

The paramount function of $\mathrm{PBMC}$ is to provide trophic support for endometrium to 
Fig 1. Hypothesis of interactive effect of PBMC immunotherapy and luteal progesterone level for implantation and pregnancy success. After intrauterine administration PBMC, the Th1 / Th2 balance towards Th1 tends to ensure ignition shift by secreting pro-inflammatory cytokines and several growth factors primarily VEGF inducing vascularization in one hand into endometrium to prepare for embryo invasion embryo and in the other hand into CL in order to increase luteal progesterone level (LPL). All these immune-endocrine factors are limited in closest communication circle with mutual interactive modulation to ensure the embryo implantation. Thereafter, an increased LPL can induce immunomodulation by promoting $\mathrm{T}$ cells differentiation into Treg and secreting PIBF as an immunosuppressor factor that promotes the Th1/Th2 balance to Th2 anti-inflammatory system ensuring immunotolerance of allograft "embryo". Thus, Th2 cytokines secretion involved in CL maturation can eventually to increase more LPL required for pregnancy maintain. CL: Corpus Luteum; LPL: Luteal progesterone level; I. S (1): Immune System (Pro-inflammatory); I. S (2): Immune System (Anti-inflammatory); PBMC: Peripheral Blood Mononuclear Cells; End: Endometrium, Emb: embryo; Vasc: Vascularization; PIBF: Progesterone induced blocked factor; VEGF: Vascular Epidermal Growth Factor, Treg: Lymphocyte T regulator.

be decidualized and for formation of dense vascular network in CL to produce more P4 that is essential for pregnancy maintain. However, perturbations of immune-endothelial cell crosstalk within the ovary during the peri-conceptional period are likely to be pivotal in luteal insufficiency in women. This issue could provide more therapeutic trends to enhance luteal function through the targeting of immune system.

\section{Conclusion}

This immunotherapeutic strategy based on PBMC intrauterine administration suggests that embryo implantation is controlled by maternal immune cells in utero and this treatment is showed its efficiency for RIF patients doubling their clinical outcomes with significant increase of LPL. This issue demonstrated that immunotherapy had positive effect on luteal P4 synthesis during implantation which acted dually on homing maternal immune system into endometrium in order to maintain pregnancy. This non-invasive and much less expensive treatment than the multiplication of IVF attempts could be proposed as part of ART to patients since their second implantation failure or even for patients with RPL or RM who are directly redirected to be treated with P4 supplementation or other anti-inflammatory treatments. Nevertheless, this issue needs an eventual researches and clinical investigations.

\section{Acknowledgments}

The authors acknowledge the help and expertise of the research team of Anfa Fertility Center at Embryology laboratory and LABOMAC clinical laboratory.

\section{References}

1. Allen WM, Corner GW. Physiology of the corpus luteum. Am J Physiol. 1929; 88: $340-346$. 
2. Allen E, Doisy EA. An ovarian hormone: Preliminary report on its localization, extraction and partial purification, and action in test animals. JAMA. 1983; 250:2681-2683.

3. Csapo AI, Pulkkinen MO, Kaihola HL. The effect of luteectomy-induced progesterone-withdrawal on the oxytocin and prostaglandin response of the first trimester pregnant human uterus. Prostaglandins. 1973; 4:421—429.

4. Hansen P. Regulation of uterine immune function by progesterone-lessons from the sheep. J Reprod Immunol. 1998; 40:63-79.

5. Mannel DN, Falk W, Yron I. Inhibition of murine cytotoxic T cell responses by progesterone. Immunol Lett. 1990; 26:89 - 94.

6. Yie SM, Li LH, Li GM, Xiao R, Librach CL. Progesterone enhances HLA-G gene expression in JEG-3 choriocarcinoma cells and human cytotrophoblasts in vitro. Hum Reprod. 2006; 21:46-51.

7. Brierley J, Clark DA. Characterization of hormone-dependent suppressor cells in the uterus of mated and pseudopregnant mice. J Reprod Immunol. 1987; $10: 201-217$.

8. Mor G, Cardenas I, Abrahams V, Guller S. Inflammation and pregnancy: the role of the immune system at the implantation site. Ann New York Acad Sci. 2011; 1221(1): 80-87.

9. Szekeres-Bartho J, Kilar F, Falkay G, Csernus V, Torok A, Pacsa AS. The mechanism of the inhibitory effect of progesterone on lymphocyte cytotoxicity: I. Progesterone-treated lymphocytes release a substance inhibiting cytotoxicity and prostaglandin synthesis. Am J Reprod Immunol Microbiol. 1985; 9:15-18.

10. Szekeres-Bartho J, Barakonyi A, Par G, Polgar B, Palkovics T, Szereday L. Progesterone as an immunomodulatory molecule. Int Immunopharmacol. 2001; 1:1037-1048.

11. Yie SM, Xiao R, Librach CL. Progesterone regulates HLA-G gene expression through a novel progesterone response element. Hum Reprod. 2006; $21: 2538-2544$.

12. Van Vollenhoven RF, McGuire JL. Estrogen, progesterone, and testosterone: Can they be used to treat autoimmune diseases? Cleve Clin J Med. 1994; 61:276 — 284 .

13. Hashii K, Fujiwara H, Yoshioka S, Kataoka N, Yamada S, Hirano T, et al. Peripheral blood mononuclear cells stimulate progesterone production by luteal cells derived from pregnant and non-pregnant women: possible involvement of interleukin-4 and interleukin-10 in corpus luteum function and differentiation. Hum Reprod. 1998; 13:2738-2744.

14. Hardy DB, Janowski B, Corey DR, Mendelson CR. Progesterone receptor plays a major antiinflammatory role in human myometrial cells by antagonism of nuclear factor-kappaB activation of cyclooxygenase 2 expression. Mol Endocrinol. 2006; $20: 2724-2733$.

15. Carlino C, Stabile H, Morrone S, Bulla R, Soriani A, Agostinis C, et al. Recruitment of circulating NK cells through decidual tissues: A possible mechanism controlling NK cell accumulation in the uterus during early pregnancy. Blood. 2008; 111:3108 - 3115. 
16. Lei K, Chen L, Georgiou EX, Sooranna SR, Khanjani S, Brosens JJ, et al. Progesterone Acts via the Nuclear Glucocorticoid Receptor to Suppress IL-1 $\beta$-Induced COX-2 Expression in Human Term Myometrial Cells. PLoS ONE. 2012; 7(11): e50167. https://doi.org/10.1371/journal.pone.0050167

17. Lei K, Georgiou EX, Chen L, Yulia A, Sooranna SR, Brosens JJ, et al. Progesterone and the repression of myometrial inflammation: the roles of MKP-1 and the AP-1 system. Mol Endocrinol. 2015; 29(1):1454-1467. https://doi:10.1210/me.2015-1122

18. Piccinni MP, Giudizi MG, Biagiotti R, Beloni L, Giannarini L, Sampognaro S, et al. Progesterone favors the development of human $\mathrm{T}$ helper cells producing Th2-type cytokines and promotes both IL-4 production and membrane CD30 expression in established Th1 cell clones. J Immunol. 1995; 155(1):128-133.

19. Choi BC, Polgar K, Xiao L, Hill J. Progesterone inhibits in-vitro embryotoxic Th1 cytokine production to trophoblast in women with recurrent pregnancy loss. Hum Reprod. 2000; 15 Suppl 1:46-59.

20. Fujiwara H, Araki Y, Imakawa K, Saito S, Daikoku T, Shigeta M, et al. Dual Positive Regulation of Embryo Implantation by Endocrine and Immune Systems? Step-by-Step Maternal Recognition of the Developing Embryo. Am J Reprod Immunol. 2016; 75(3):281—289. https://doi:10.1111/aji.12478

21. Szekeres-Bartho J, Balasch J. Progestagen therapy for recurrent miscarriage. Hum Reprod Update. 2008; 14(1):27-35.

22. Madkour A, Bouamoud N, Louanjli N, Kaarouch I, Copin H, Benkhalifa M, et al. Intrauterine insemination of cultured peripheral blood mononuclear cells prior to embryo transfer improves clinical outcome for patients with repeated implantation failures. Zygote. 2016; 24(1):58-69. https://doi:10.1017/S0967199414000719

23. Yoshioka S, Fujiwara H, Nakayama T, Kosaka K, Mori T, Fujii S. Intrauterine administration of autologous peripheral blood mononuclear cells promotes implantation rates in patients with repeated failure of IVF-embryo transfer. Hum Reprod. 2006; 21(12):3290 - 3294.

24. Okitsu O, Kiyokawa M, Oda T, Miyake K, Sato Y, Fujiwara H. Intrauterine administration of autologous peripheral blood mononuclear cells increases clinical pregnancy rates in frozen/thawed embryo transfer cycles of patients with repeated implantation failure. J Reprod Immunol. 2011; 92(1-2):82--87. https://doi:10.1016/j.jri.2011.07.001

25. Makrigiannakis A, BenKhalifa M, Vrekoussis T, Mahjub S, Kalantaridou SN, Gurgan T. Repeated implantation failure: a new potential treatment option. Eur J Clin Invest. 2015; 45(4):380 - 384. https://doi:10.1111/eci.12417

26. Yu N, Zhang B, Xu M, Wang S, Liu R, Wu J, et al. Intrauterine administration of autologous peripheral blood mononuclear cells (PBMCs) activated by HCG improves the implantation and pregnancy rates in patients with repeated implantation failure: a prospective randomized study. Am J Reprod Immunol. 2016; 76(3):212-216. https://doi:10.1111/aji.12542

27. Li S, Wang J, Cheng Y, Zhou D, Yin T, Xu W, et al. Intrauterine administration of hCG-activated autologous human peripheral blood mononuclear cells (PBMC) promotes live birth rates in frozen/thawed embryo transfer cycles of patients with repeated implantation failure. J Reprod Immunol. 2017; 119:15-22. https://doi:10.1016/j.jri.2016.11.006 
28. Coughlan C, Ledger W, Wang Q, Liu F, Demirol A, Gurgan T, et al. Recurrent implantation failure: Definition and management. Reprod Biomed Online. 2014; 28(1):14-38. https://doi:10.1016/j.rbmo.2013.08.011

29. Sharma S, Majumdar A. Determining the Optimal Duration of Progesterone Supplementation prior to Transfer of Cryopreserved Embryos and Its Impact on Implantation and Pregnancy Rates: A Pilot Study. Int J Reprod Med. 2016; 2016:7128485.

30. Kalinka J, Radwan M. The impact of dydrogesterone supplementation on serum cytokine profile in women with threatened abortion. Am J Reprod Immunol. 2006; 55(2):115-121.

31. Raghupathy R, Al Mutawa E, Makhseed M, Azizieh F, Szekeres-Bartho J. Modulation of cytokine production by dydrogesterone in lymphocytes from women with recurrent miscarriage. BJOG. 2005; 112(8):1096-1101.

32. Al-Sebai MA, Kingsland CR, Diver M, Hipkin L, McFadyen IR. The role of a single progesterone measurement in the diagnosis of early pregnancy failure and the prognosis of fetal viability. Br J Obstet Gynaecol. 1995; 102(5):364-369.

33. Mitra A, Mandana B. Application of gel-based proteomic technique in female reproductive investigations. J Hum Reprod Sci. 2015; 8(1):18-24. https://doi:10.4103/0974-1208

34. Yu N, Yan W, Yin T, Wang Y, Guo Y, Zhou D, et al. HCG-activated human peripheral blood mononuclear cells (PBMC) promote trophoblast cell invasion. PLoS One. 2015; 10(6):e0125589. https://doi:10.1371/journal.pone.0125589

35. Hussain M, El-Hakim S, Cahill DJ. Progesterone supplementation in women with otherwise unexplained recurrent miscarriages. J Hum Reprod Sci. 2012; 5(3):248 - 251. https://doi:10.4103/0974-1208.106335

36. Navot D, Anderson TL, Droesch K, Scott RT, Kreiner D, Rosenwaks Z. Hormonal manipulation of endometrial maturation. J Clin Endocrinol Metab. $1989 ; 68(4): 801-807$.

37. Joachim R, Zenclussen AC, Polgar B, Douglas AJ, Fest S, Knackstedt M, et al. The progesterone derivative dydrogesterone abrogates murine stress-triggered abortion by inducing a Th2 biased local immune response. Steroids. 2003; 68(10-13):931-940.

38. Georgiou EX, Lei K, Lai PF, Yulia A, Herbert BR, Castellanos M, et al. The study of progesterone action in human myometrial explants. Mol Hum Reprod. 2016; 22(8):577-589. https://doi:10.1093/molehr/gaw037

39. Omar MH, Mashita MK, Lim PS, Jamil MA. Dydrogesterone in threatened abortion: Pregnancy outcome. J Steroid Biochem Mol Biol. 2005; 97(5):421—425.

40. Coomarasamy A, Truchanowicz EG, Rai R. Does first trimester progesterone prophylaxis increase the live birth rate in women with unexplained recurrent miscarriages?. BMJ. 2011; 342:d1914. https://doi:10.1136/bmj.d1914

41. Care AS, Diener KR, Jasper MJ, Brown HM, Ingman WV, Robertson SA. Macrophages regulate corpus luteum development during embryo implantation in mice. J Clin Invest. 2013; 23(8):3472 — 3487. https://doi:10.1172/JCI60561 
42. Arceci RJ, Shanahan F, Stanley ER, Pollard JW. Temporal expression and location of colony-stimulating factor $1(\mathrm{CSF}-1)$ and its receptor in the female reproductive tract are consistent with CSF-1-regulated placental development. Proc Natl Acad Sci U S A. 1989; 86(22):8818-8822.

43. Kizuka F, Tokuda N, Takagi K, Adachi Y, Lee L, Tamura I, et al. Involvement of Bone Marrow-Derived Vascular Progenitor Cells in Neovascularization During Formation of the Corpus Luteum in Mice. Biol Reprod. 2012; 87(3):55. https://doi:10.1095/biolreprod.112.099960 


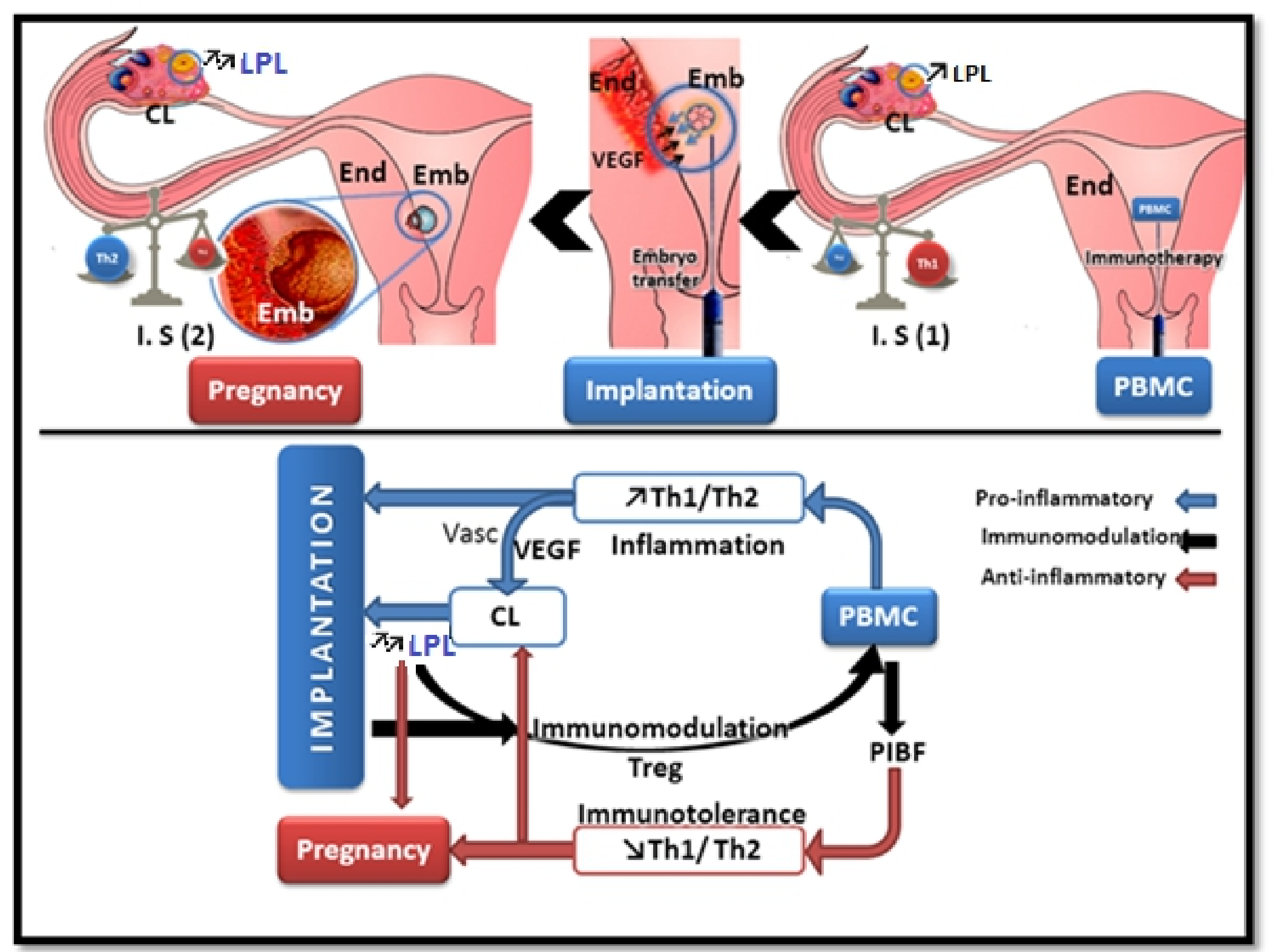

\section{Figure}

